\title{
Guillain-Barre syndrome in 220 patients with COVID-19
}

\author{
Josef Finsterer $^{1 *}$ and Fulvio A. Scorza ${ }^{2}$ (B)
}

\begin{abstract}
This review summarises and discusses recent findings concerning the pathophysiology, clinical presentation, diagnosis, treatment, and outcome of SARS-CoV-2-associated Guillain-Barre syndrome (SC2-GBS). By the end of December 2020, at least 220 patients with SC2-GBS have been published in 95 papers. SC2-GBS is most likely secondary due to an immune reaction against SARS-CoV-2 since the virus has not been found in the CSF of any SC2-GBS patient so far reported. SC2-GBS occurs in each age group and does not differ from non-SC2-GBS regarding clinical presentation and treatment, but the outcome of SC2-GBS is worse compared to non-CS2-GBS patients, and the prevalence/incidence of GBS most likely increased since the outbreak of the pandemic. Early diagnosis of SC2-GBS is warranted to apply appropriate treatment in due time and to improve the overall outcome from the infection.
\end{abstract}

Keywords: SARS-CoV-2, COVID-19, Guillain-Barre syndrome, Nerve conduction, Immunoglobulins

\section{Introduction}

Since the outbreak of the pandemic by the SARS-CoV-2 virus, it became rapidly obvious that the virus not only causes lung disease (COVID-19) but affects other organs as well, particularly the central and peripheral nervous system (PNS, CNS), the kidneys, the intestines, and the heart [1-3]. The most disabling PNS disorder is polyradiculitis (polyradiculoneuritis, Guillain-Barre syndrome (GBS)) [4]. GBS comprises a number of subtypes which include acute, inflammatory, demyelinating neuropathy (AIDP) (classic type), acute, motor, axonal neuropathy (AMAN), acute, motor and sensory, axonal neuropathy (AMSAN), Miller-Fisher syndrome (MFS), polyneuritis cranialis (PNC), the pharyngeal, cervical, and brachial (PCB) variant, and Bickerstaff encephalitis (BFE) [5]. GBS is usually diagnosed according to the Brighton criteria if there is bilateral, progressive, flaccid lower > upper limb paraparesis, if tendon reflexes in weak limbs are diminished, if the disease course is monophasic and if time between onset and nadir ranges from $12 \mathrm{~h}$ to 28

\footnotetext{
*Correspondence: fifigs1@yahoo.de

${ }^{1}$ Klinik Landstrasse, Messerli Institute, Postfach 20, 1180 Vienna, Austria

Full list of author information is available at the end of the article
}

days, if cerebrospinal fluid (CSF) investigations reveal a cell count $<50$ cells $/ \mu \mathrm{L}$, if CSF protein is elevated (dissociation cyto-albuminque (DCA)), and if nerve conduction studies show a demyelinating lesion of motor nerves (AIDP), an axonal lesion of motor nerves (AMAN), or an axonal lesion of motor and sensory nerves (AMSAN) [5]. MFS is diagnosed if there is acute onset ophthalmoplegia, areflexia, ataxia, and DCA. PNC is diagnosed in case of a lesion of a single or multiple cranial nerves and DCA. $\mathrm{PCB}$ is diagnosed if there is progressive dysphagia, dysphonia, upper limb weakness, and DCA [5]. BFE is diagnosed if there are pyramidal signs and impaired consciousness in addition to MFS. In the early stages of GBS, upper or lower limb paraplegia with preserved tendon reflexes may occur [5]. There can be even hyperreflexia if the pyramidal tract is involved. All GBS subtypes occur in the setting of a preceding viral or bacterial infection. The type of preceding infection largely determines the subtype and clinical course of GBS. This systematised review summarises and discusses recent findings and future perspectives concerning the pathophysiology, clinical presentation, diagnosis, treatment, and outcome of SARSCoV-2-associated GBS (SC2-GBS). 


\section{Methods}

A systematised literature search in the databases PubMed and Google Scholar using the search terms "neuropathy," "Guillain Barre syndrome," "polyradiculitis," "AIDP," "AMAN," “AMSAN," "Miller-Fisher syndrome," "polyneuritis cranialis," and "Bickerstaff encephlaitis," in combination with "SARS-CoV-2," "COVID-19," and "coronavirus" was conducted. Additionally, reference lists were checked for further articles meeting the search criteria. Included were only original articles detailing individual patients' data (age, sex, latency between onset of COVID-19 and SC2-GBS, GBS subtype, results of CSF investigations, treatment, and outcome) and written in English, French, Spanish, Italian, or German, Excluded from data analysis were reviews, abstracts, proceedings, and editorials as well as original studies not specifying individual patients' data (Fig. 1).

\section{Main text}

By the end of December 2020, at least 220 patients with SC2-GBS have been published in 95 papers (Tables 1 and 2). Age of these patients (reported in $n=215$ ) ranged from 8-94 years (Table 1). Gender (reported in $n$ = 213) was male in 146 and female in 67 (Tables 1 and 2 ). Onset (reported in $n=165$ ) was identified after/together with/before onset of non-neurological COVID-19 manifestations in 156/3/6 patients (Tables 1 and 2). Latency between onset of COVID-19 and GBS $(n=194)$ ranged from - 10 to 90 days. One patient remained asymptomatic. The GBS subtype (reported in $n=152$ ) was identified as AIDP $(n=118)$, AMAN $(n=13)$, AMSAN $(n=11)$, MFS $(n=7)$, PNC $(n=2)$, the PCB variant $(n=1)$, and $\operatorname{BFE}(n=0)$. SARS-CoV-2 was not detected in the CSF in any of the patients (Table 1). Therapy of GBS (reported in $n=215$ ) comprised intravenous immunoglobulins (IVIG) $(n=191)$, plasmapheresis $(n=15)$, steroids $(n=2)$, or no therapy $(n=7)$ (Tables 1 and 2). Forty-one patients required artificial ventilation (Tables 1 and 2). Outcome (reported in $n=$ $168)$ was assessed as complete recovery $(n=37)$, partial recovery $(n=119)$, or death $(n=12)$ (Tables 1 and 2$)$. No studies about factors determining the outcome of SC2-GBS subtypes were identified.

\section{Discussion}

This systematic review shows that SC2-GBS is not due to a direct attack of the virus but rather due to an immunological reaction to the virus. It also shows that the number of reports about SC2-GBS is increasing and that the outcome is worse compared to non-SC2-GBS [6].

Though the number of cases with SC2-GBS is increasing suggesting that the overall prevalence of GBS has increased since the outbreak of the pandemic, there are conflicting results concerning this matter. In a UK study of 47 SC2-GBS patients, the prevalence of GBS did not increase between March 2020 and May 2020 as compared to the years 2016-2019 [6]. On the contrary, a

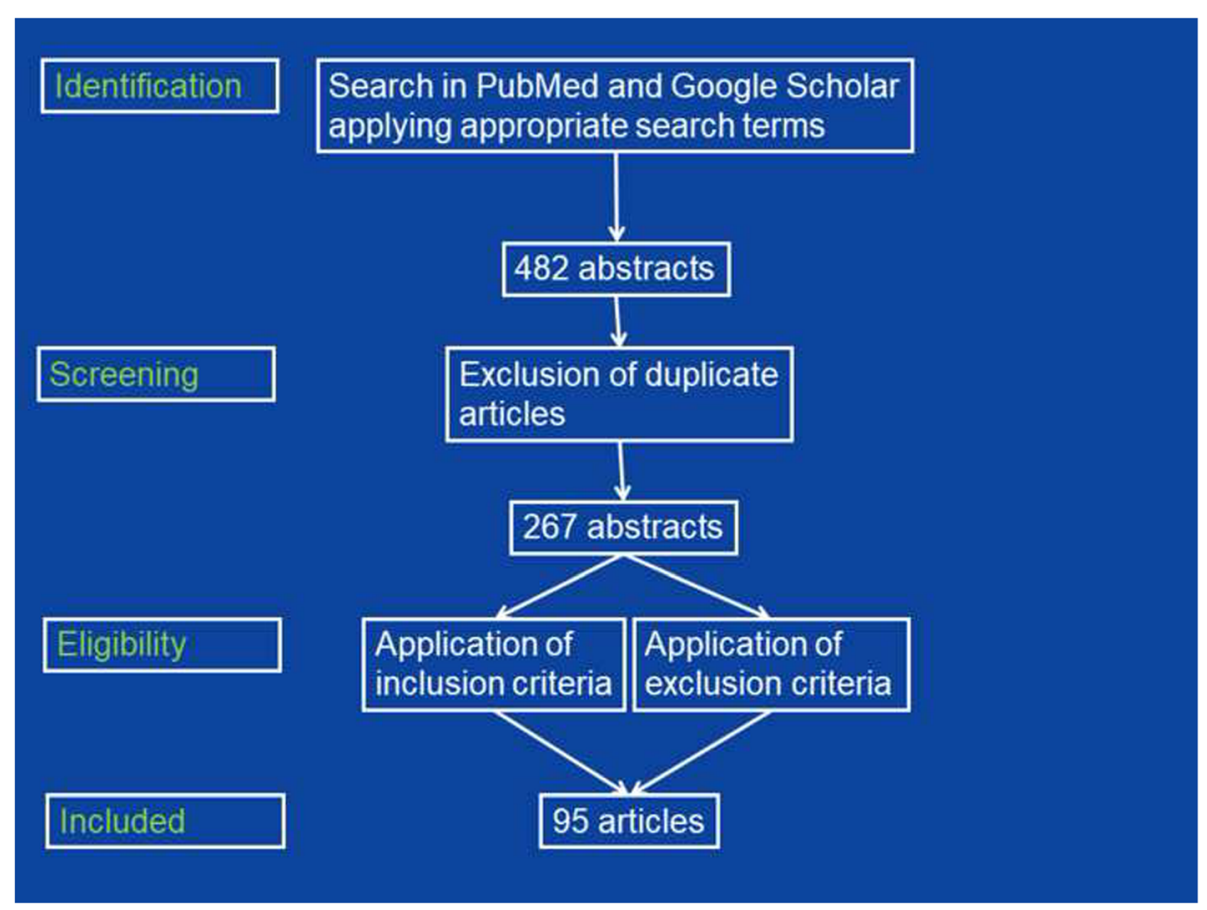

Fig. 1 Flow chart detailing the search protocol and the results after application of inclusion and exclusion criteria 
Table 1 Patients with SARS-CoV-2 associated polyradiculitis as reported by the end of December 2020

\begin{tabular}{|c|c|c|c|c|c|c|c|c|c|c|}
\hline Age (years) & Sex & Onset & LOO (days) & Subtype & $\mathrm{CIC}$ & CM & IVIG & AV & Recovery & Country \\
\hline$\overline{61}$ & $f$ & $B$ & 9 & AIDP & $\mathrm{nr}$ & No & Yes & No & Yes & China \\
\hline 65 & $\mathrm{~m}$ & A & 9 & AMSAN & nd & DM & Yes & No & $\mathrm{nr}$ & Iran \\
\hline 54 & $\mathrm{~m}$ & A & 8 & AIDP & $\mathrm{nr}$ & No & Yes & Yes & Yes & USA \\
\hline 70 & $f$ & A & 23 & AIDP & nd & No & Yes & Yes & $\mathrm{nr}$ & Italy \\
\hline 66 & $f$ & A & 7 & AIDP & No & $\mathrm{nr}$ & Yes & Yes & Yes & Italy \\
\hline 54 & $f$ & A & 21 & AIDP & nd & No & Yes & No & Yes & Germany \\
\hline 70 & $f$ & A & 3 & AMSAN & No & RA & Yes & No & Partial & Morocco \\
\hline 20 & $\mathrm{~m}$ & A & 5 & AMAN & nd & No & Yes & No & Yes & India \\
\hline 71 & $\mathrm{~m}$ & A & 4 & AIDP & No & AHT, AAR, LC & Yes & Yes & Death & Italy \\
\hline 64 & $\mathrm{~m}$ & A & 11 & AIDP & nd & No & Yes & Yes & $\mathrm{nr}$ & France \\
\hline $\mathrm{nr}$ & $\mathrm{nr}$ & A & 7 & AIDP & No & $\mathrm{nr}$ & Yes & No & Partial & Italy \\
\hline $\mathrm{nr}$ & $\mathrm{nr}$ & A & 10 & AIDP & No & $\mathrm{nr}$ & Yes & No & Yes & Italy \\
\hline $\mathrm{nr}$ & $\mathrm{nr}$ & A & 10 & AMAN & No & $\mathrm{nr}$ & Yes & Yes & Partial & Italy \\
\hline $\mathrm{nr}$ & $\mathrm{nr}$ & A & 5 & AMAN & No & $\mathrm{nr}$ & Yes & No & Partial & Italy \\
\hline $\mathrm{nr}$ & $\mathrm{nr}$ & A & 7 & AMAN & No & $\mathrm{nr}$ & Yes, PE & No & $\mathrm{nr}$ & Italy \\
\hline 50 & $\mathrm{~m}$ & A & 3 & MFS, PNC & No & No & Yes & No & Yes & Spain \\
\hline 39 & $\mathrm{~m}$ & A & 3 & MFS, PNC & No & No & No & No & Yes & Spain \\
\hline 61 & $\mathrm{~m}$ & A & 10 & MFS & No & No & $S$ & No & Yes & Spain \\
\hline 76 & $f$ & A & 8 & GBS (no NCS) & nd & No & No & $\mathrm{nr}$ & Death & Spain \\
\hline$\sim 75$ & $\mathrm{~m}$ & B & 10 & AIDP & No & No & Yes & No & Yes & Swiss \\
\hline 43 & $\mathrm{~m}$ & A & 10 & AIDP & $\mathrm{nr}$ & $\mathrm{nr}$ & Yes & No & Yes & Spain \\
\hline 64 & $\mathrm{~m}$ & A & 23 & AIDP & No & $\mathrm{nr}$ & Yes & No & Yes & France \\
\hline 72 & $\mathrm{~m}$ & A & 7 & AIDP & No & $\mathrm{AHT}, \mathrm{CHD}, \mathrm{AL}$ & Yes & Yes & Partial & USA \\
\hline$\sim 65$ & $\mathrm{~m}$ & A & 17 & AIDP & No & No & Yes & No & Yes & Italy \\
\hline 67 & $f$ & A & 10 & $\mathrm{nr}$ & No & Breast cancer & PE & Yes & Partial & USA \\
\hline 54 & $\mathrm{~m}$ & A & 14 & AIDP & nd & $\mathrm{nr}$ & Yes & No & Partial & USA \\
\hline 43 & $\mathrm{~m}$ & A & 21 & AIDP & No & $\mathrm{nr}$ & Yes & No & Yes & France \\
\hline 71 & $f$ & A & 10 & AIDP & No & $\mathrm{nr}$ & Yes & No & Partial & France \\
\hline 36 & $\mathrm{~m}$ & A & 4 & MFS & $\mathrm{nr}$ & $\mathrm{nr}$ & Yes & No & Yes & USA \\
\hline 55 & $\mathrm{~m}$ & A & 20 & AIDP & No & $\mathrm{nr}$ & Yes & Yes & Partial & Italy \\
\hline 60 & $\mathrm{~m}$ & A & 3 & AMSAN & No & $\mathrm{nr}$ & Yes & Yes & Partial & Italy \\
\hline 58 & $\mathrm{~m}$ & $A B$ & 0 & AIDP & No & No & Yes & No & Partial & Canada \\
\hline 52 & $f$ & A & 15 & AIDP & No & $\mathrm{nr}$ & Yes & No & Partial & Swiss \\
\hline 63 & $f$ & A & 7 & AIDP & $\mathrm{nr}$ & $\mathrm{nr}$ & Yes & No & Yes & Swiss \\
\hline 61 & $f$ & A & 22 & AIDP & No & $\mathrm{nr}$ & Yes & No & Partial & Swiss \\
\hline 53 & $f$ & B & $\mathrm{nr}$ & AIDP & No & No & $P E$ & No & Partial & Turkey \\
\hline 51 & $f$ & A & 14 & MFS & $\mathrm{nr}$ & $\mathrm{nr}$ & Yes & No & Partial & Spain \\
\hline 56 & $f$ & A & 15 & AIDP & No & $\mathrm{nr}$ & Yes & Yes & Partial & Spain \\
\hline 68 & $\mathrm{~m}$ & A & 14 & AIDP & $\mathrm{nr}$ (ASPC) & $\mathrm{nr}$ & Yes, PE & Yes & Partial & Austria \\
\hline 55 & $f$ & A & 14 & AIDP & $\mathrm{nr}$ & No & Yes & Yes & Partial & Spain \\
\hline 53 & $\mathrm{~m}$ & A & 24 & AIDP & No & No & Yes & No & Yes & Netherlands \\
\hline 57 & $\mathrm{~m}$ & A & 6 & AIDP & No & AHT, psoriasis & Yes & Yes & Partial & UK \\
\hline 21 & $\mathrm{~m}$ & A & 16 & AIDP & $\mathrm{nr}$ & $\mathrm{AHT}, \mathrm{DM}$ & $P E$ & No & Yes & USA \\
\hline 41 & $\mathrm{~m}$ & A & 10 & AIDP & $\mathrm{nr}$ & DM & Yes & No & Partial & Iran \\
\hline
\end{tabular}


Table 1 Patients with SARS-CoV-2 associated polyradiculitis as reported by the end of December 2020 (Continued)

\begin{tabular}{|c|c|c|c|c|c|c|c|c|c|c|}
\hline Age (years) & Sex & Onset & LOO (days) & Subtype & $\mathrm{CIC}$ & CM & IVIG & AV & Recovery & Country \\
\hline 38 & $m$ & A & 16 & AIDP & $\mathrm{nr}$ & AHT & $P E$ & No & Yes & Iran \\
\hline 14 & $f$ & A & $\mathrm{nr}$ & GBS & $\mathrm{nr}$ & No & Yes & No & Yes & Iran \\
\hline 49 & $\mathrm{~m}$ & A & 14 & AIDP & No & No & Yes & No & Yes & UK \\
\hline 68 & $\mathrm{~m}$ & A & 5 & AIDP & $\mathrm{nr}$ & AHT, HLP & Yes & No & Yes & Italy \\
\hline 11 & $\mathrm{~m}$ & A & 21 & AIDP & $\mathrm{nr}$ & No & Yes & No & Yes & Saudi \\
\hline 15 & $\mathrm{~m}$ & A & $\mathrm{nr}$ & AMAN & No & No & Yes & No & Partial & Brazil \\
\hline 72 & $\mathrm{~m}$ & A & 18 & AIDP & No & $\mathrm{nr}$ & Yes & Yes & Partial & Italy \\
\hline 72 & $\mathrm{~m}$ & A & 30 & AIDP & No & $\mathrm{nr}$ & Yes & Yes & Partial & Italy \\
\hline 49 & $f$ & A & 14 & AIDP & No & $\mathrm{nr}$ & Yes & No & Partial & Italy \\
\hline 94 & $\mathrm{~m}$ & A & 33 & AIDP & $\mathrm{nr}$ & $\mathrm{nr}$ & S & No & Partial & Italy \\
\hline 76 & $\mathrm{~m}$ & A & 22 & AIDP & No & $\mathrm{nr}$ & Yes & Yes & Partial & Italy \\
\hline 64 & $\mathrm{~m}$ & A & $\mathrm{nr}$ & GBS? & $\mathrm{nr}$ & DM & Yes & Yes & Yes & Japan \\
\hline 77 & $\mathrm{~m}$ & A & $\mathrm{nr}$ & AIDP & $\mathrm{nr}$ & AHT, HLP & Yes & No & Yes & Spain \\
\hline 58 & $f$ & A & 6 & AIDP & No & $\mathrm{nr}$ & PE & No & Yes & USA \\
\hline 56 & $f$ & A & 7 & AIDP & No & AHT, thyroxin $\downarrow$ & $\mathrm{nr}$ & $\mathrm{nr}$ & Partial & Germany \\
\hline 61 & $f$ & A & 14 & AMAN & No & AHT, HLP & PE & No & Yes & USA \\
\hline 75 & $\mathrm{~m}$ & A & $\mathrm{nr}$ & $\mathrm{nr}$ & No & spinal trauma & Yes & No & Yes & USA \\
\hline 37 & $\mathrm{nr}$ & A & 10 & $\mathrm{nr}$ & $\mathrm{nr}$ & $\mathrm{nr}$ & $\mathrm{nr}$ & $\mathrm{nr}$ & $\mathrm{nr}$ & Belgium \\
\hline 60 & $f$ & A & 22 & $\mathrm{nr}$ & $\mathrm{nr}$ & Migraine & Yes & No & Partial & USA \\
\hline \multirow[t]{2}{*}{$\varnothing 57$} & $33 \mathrm{~m}$ & $\mathrm{nr}$ & $0-37$ & $\mathrm{nr}$ & $\mathrm{nr}$ & $\mathrm{nr}$ & Yes, $n=46$ & $\mathrm{nr}$ & Death, $n=1$ & UK, $n=47$ \\
\hline & & & & & & & $\mathrm{PE}, n=1$ & & $\mathrm{nr}, n=46$ & \\
\hline 51 & $\mathrm{~m}$ & A & 12 & AIDP & No & $\mathrm{nr}$ & Yes & Yes & Partial & Germany \\
\hline 34 & $\mathrm{~m}$ & A & 4 & PNC & $\mathrm{nr}$ & Strabism & Yes & No & Partial & USA \\
\hline 71 & $f$ & A & Days & PNC & $\mathrm{nr}$ & AHT & No & No & Partial & USA \\
\hline 65 & $\mathrm{~m}$ & A & 3 & AIDP & $\mathrm{nr}$ & No & Yes & No & Yes & Germany \\
\hline 74 & $f$ & A & $\mathrm{nr}$ & AIDP & No & Lymphoma & Yes & No & Yes & Spain \\
\hline 49 & $\mathrm{~m}$ & A & 14 & MFS & No & Crohn's disease & Yes & Yes & partial & USA \\
\hline 65 & $f$ & A & $\mathrm{nr}$ & AIDP & $\mathrm{nr}$ & Fibromyalgia & Yes & Yes & Death & Italy \\
\hline 12 & $\mathrm{~m}$ & A & 7 & $\mathrm{nr}$ & $\mathrm{nr}$ & No & Yes & Yes & Death & Tanzania \\
\hline 88 & $f$ & A & 2 & AMSAN & $\mathrm{nr}$ & $\mathrm{nr}$ & PE & Yes & Partial & Iran \\
\hline 47 & $\mathrm{~m}$ & A & 7 & AMSAN & $\mathrm{nr}$ & $\mathrm{nr}$ & PE & Yes & Death & Iran \\
\hline 58 & $\mathrm{~m}$ & A & 9 & AMSAN & $\mathrm{nr}$ & $\mathrm{nr}$ & Yes, PE & Yes & Death & Iran \\
\hline 54 & $\mathrm{~m}$ & A & 3 & $\mathrm{nr}$ & $\mathrm{nr}$ & GBS, DN & Yes & No & Yes & USA \\
\hline 57 & $\mathrm{~m}$ & A & $\mathrm{nr}$ & AMAN & $\mathrm{nr}$ & $\mathrm{nr}$ & Yes & No & $\mathrm{nr}$ & Italy \\
\hline 37 & $\mathrm{~m}$ & A & 14 & AIDP & $\mathrm{nr}$ & $\mathrm{nr}$ & Yes & Yes & Partial & Iran \\
\hline 41 & $\mathrm{~m}$ & A & 10 & AIDP & No & $\mathrm{nr}$ & Yes & No & Yes & Guinea \\
\hline 76 & $\mathrm{~m}$ & A & 7 & AIDP & No & Cardiomyopathy & Yes & No & Partial & France \\
\hline \multirow[t]{2}{*}{$\varnothing 59.2$} & $22 \mathrm{~m}$ & A & $16-35$ & AIDP, $n=23$ & $\mathrm{nr}$ & Several & $n=25$ & $n=5$ & Partial & UK, $n=30$ \\
\hline & & & & AMAN, $n=2$ & & & $\mathrm{PE}, n=2$ & & & \\
\hline 44 & $\mathrm{~m}$ & A & $\mathrm{nr}$ & $\mathrm{nr}$ & $\mathrm{nr}$ & AHT, asthma & Yes & No & Yes & USA \\
\hline 54 & $f$ & A & 20 & AMAN & $\mathrm{nr}$ & Asthma & No & No & Partial & Japan \\
\hline 55 & $f$ & A & 11 & AMSAN & $\mathrm{nr}$ & Lung disease & Yes & Yes & Death & Iran \\
\hline 8 & $\mathrm{~m}$ & B & $\mathrm{nr}$ & AIDP & No & No & Yes & Yes & Partial & USA \\
\hline 65 & $\mathrm{~m}$ & A & 14 & AIDP & $\mathrm{nr}$ & $\mathrm{nr}$ & Yes & No & Partial & Iran \\
\hline
\end{tabular}


Table 1 Patients with SARS-CoV-2 associated polyradiculitis as reported by the end of December 2020 (Continued)

\begin{tabular}{|c|c|c|c|c|c|c|c|c|c|c|}
\hline Age (years) & Sex & Onset & LOO (days) & Subtype & $\mathrm{CIC}$ & CM & IVIG & AV & Recovery & Country \\
\hline 70 & $f$ & A & 90 & $\mathrm{nr}$ & $\mathrm{nr}$ & RSD & Yes & No & Yes & USA \\
\hline 55 & $f$ & A & 10 & AMAN & $\mathrm{nr}$ & $\mathrm{DM}, \mathrm{AHT}$ & Yes & No & Partial & India \\
\hline 72 & $\mathrm{~m}$ & A & 6 & AIDP & $\mathrm{nr}$ & AHT & Yes & Yes & Death & India \\
\hline 55 & $\mathrm{~m}$ & A & 7 & AMSAN & $\mathrm{nr}$ & $\mathrm{DM}, \mathrm{AHT}, \mathrm{Rl}$ & Yes & No & Partial & India \\
\hline 49 & $\mathrm{~m}$ & A & 10 & AIDP & $\mathrm{nr}$ & $\mathrm{DM}, \mathrm{AHT}$ & Yes & No & Partial & India \\
\hline 53 & $\mathrm{~m}$ & A & $\mathrm{nr}$ & $\mathrm{nr}$ & $\mathrm{nr}$ & $\mathrm{nr}$ & Yes & No & Partial & Italy \\
\hline 36 & $\mathrm{~m}$ & A & 18 & AIDP & $\mathrm{nr}$ & AHT, NTX & Yes & Yes & Partial & USA \\
\hline 57 & $\mathrm{~m}$ & A & 17 & AIDP & $\mathrm{nr}$ & $\mathrm{nr}$ & Yes & No & Partial & Italy \\
\hline \multirow[t]{2}{*}{$\varnothing 53$} & $11 \mathrm{~m}$ & A & $0.5-28$ & AIDP & No, $n=4$ & $\mathrm{nr}$ & Yes, $n=15$ & $\mathrm{nr}$ & Partial, & Italy, $n=17$ \\
\hline & & & & & & & $P E, n=2$ & & Death, $n=1$ & \\
\hline 54 & $f$ & $A B$ & 0 & $\mathrm{nr}$ & No & AHT & Yes & No & Partial & Spain \\
\hline 58 & $f$ & A & 14 & $\mathrm{nr}$ & $\mathrm{nr}$ & Disc prolapse & Yes & No & Partial & USA \\
\hline 65 & $\mathrm{~m}$ & A & $\mathrm{nr}$ & AIDP & $\mathrm{nr}$ & $\mathrm{nr}$ & Yes & No & Partial & Italy \\
\hline 73 & $\mathrm{~m}$ & $A B$ & 0 & AIDP & No & $\mathrm{nr}$ & Yes & No & Partial & Italy \\
\hline 55 & $\mathrm{~m}$ & A & 20 & AIDP/MFS & No & $\mathrm{nr}$ & Yes & No & Partial & Italy \\
\hline 46 & $f$ & A & 3 & AIDP & No & $\mathrm{nr}$ & Yes & No & Partial & Italy \\
\hline 60 & $\mathrm{~m}$ & A & 20 & AMSAN & No & $\mathrm{nr}$ & Yes & No & Partial & Italy \\
\hline 63 & $f$ & A & 15 & AMSAN & $\mathrm{nr}$ & $\mathrm{nr}$ & Yes & No & Partial & Italy \\
\hline$\sim 35$ & $\mathrm{~m}$ & A & $\mathrm{nr}$ & AMAN & No & $\mathrm{nr}$ & Yes & No & Partial & UK \\
\hline 49 & $\mathrm{~m}$ & A & 11 & PCB & No & $\mathrm{AHT}$, seminoma & No & No & Partial & Italy \\
\hline 54 & $\mathrm{~m}$ & A & 4 & AIDP & $\mathrm{nr}$ & AHT, obesity & Yes & Yes & Partial & Spain \\
\hline 54 & $\mathrm{nr}$ & $\mathrm{nr}$ & $\mathrm{nr}$ & $\mathrm{nr}$ & No & AHT, HLP & Yes & Yes & Yes & Spain \\
\hline 72 & $f$ & A & 8 & AIDP & No & $\mathrm{nr}$ & Yes & Yes & Partial & Italy \\
\hline 48 & $\mathrm{~m}$ & A & 18 & AIDP & $\mathrm{nr}$ & $\mathrm{DM}$ & $P E$ & No & Partial & USA \\
\hline 46 & $\mathrm{~m}$ & A & 18 & AIDP & $\mathrm{nr}$ & $\mathrm{nr}$ & No & No & Partial & Iran \\
\hline 65 & $\mathrm{~m}$ & A & 10 & AIDP & $\mathrm{nr}$ & $\mathrm{nr}$ & Yes & No & Partial & Iran \\
\hline 66 & $f$ & B & No symptom & AIDP & $\mathrm{nr}$ & $\mathrm{nr}$ & Yes & No & Partial & Italy \\
\hline 66 & $f$ & A & 30 & AIDP & $\mathrm{nr}$ & DM, AHT, arthritis & Yes & No & Partial & Iran \\
\hline 55 & $f$ & A & 31 & AMSAN & $\mathrm{nr}$ & COPD & Yes & Yes & Death & Iran \\
\hline 14 & $f$ & A & $\mathrm{nr}$ & $\mathrm{nr}$ & $\mathrm{nr}$ & No & Yes & No & Yes & Iran \\
\hline 38 & $\mathrm{~m}$ & A & 16 & AIDP & $\mathrm{nr}$ & No & PE & No & Partial & Iran \\
\hline $20-63$ & $7 \mathrm{~m}$ & $\mathrm{nr}$ & $\mathrm{nr}$ & AIDP & $\mathrm{nr}$ & $\mathrm{nr}$ & Yes & No & Partial & UK, $n=7$ \\
\hline 65 & $\mathrm{~m}$ & A & 5 & AIDP & $\mathrm{nr}$ & $\mathrm{DM}, \mathrm{AHT}$ & Yes & Yes & Death & Sudan \\
\hline 43 & $\mathrm{~m}$ & A & 10 & AIDP & $\mathrm{nr}$ & $\mathrm{nr}$ & Yes & No & Partial & Spain \\
\hline 63 & $\mathrm{~m}$ & A & 1 & MFS & $\mathrm{nr}$ & $\mathrm{nr}$ & No & No & Partial & UK \\
\hline 61 & $\mathrm{~m}$ & A & $\mathrm{nr}$ & MFS & No & $\mathrm{nr}$ & Yes & No & Yes & Germany \\
\hline 58 & $\mathrm{~m}$ & B & $\mathrm{nr}$ & AIDP & $\mathrm{nr}$ & $\mathrm{nr}$ & Yes & Yes & Partial & UK \\
\hline 70 & $f$ & A & 15 & AMAN & $\mathrm{nr}$ & AHTt, obesity & Yes, PE & No & Partial & Italy \\
\hline
\end{tabular}

A, onset of GBS after onset of non-neurological manifestations; AAR Aortic aneurysm repair; AHT Arterial hypertension; AL Alcoholism, ASPC Antibodies for SARSCoV-2 positive in CSF; AV Artificial ventilation; B, onset of GBS before onset of non-neurological manifestations; CHD Coronary heart disease; CIC CoV2 in CSF; CM Comorbidities; DM Diabetes; $f$ Female; HLP Hyperlipidaemia; $L C$ Lung cancer; $L O O$ Latency between onset of GBS and COVID-19 respectively vice versa; $m$ Male; $n d$ Not done; $n r$ Not reported; NCS Nerve conduction study; NTX Renal transplantation; $p c$ Personal communication; PCB Pharyngeal, cervical, brachial variant of GBS; PE Plasma exchange; PNC Polyneuritis cranialis; RA Rheumatoid arthritis; RI Renal insufficiency; RSD Reflex sympathetic dystrophy; $S$ Steroids 
Table 2 Summary of findings in 220 patients with SC2-GBS

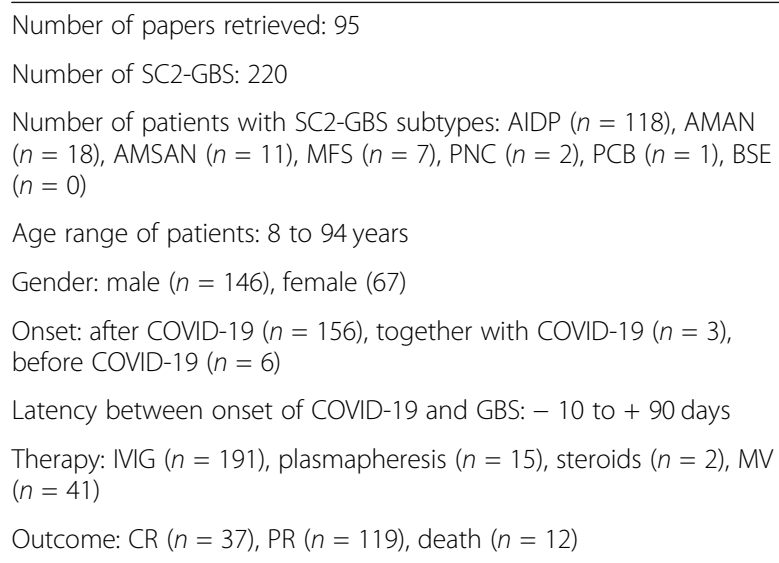

retrospective, multi-centre study from northern Italy of 34 SC2-GBS patients showed that the estimated incidence of GBS in March 2020 and April 2020 increased from $0.93 / 100000$ year in 2019 to $2.43 / 100000 /$ year in 2020 [7]. There are several reasons why SC2-GBS may be missed and why the prevalence of GBS in fact increased since onset of the pandemic. First, SC2-GBS may go undetected due to misinterpretation as increased weakness or sensory disturbances of a pre-existing neuropathy. Second, SC2-GBS may be misinterpreted as critical ill neuropathy. Third, work-up for neuropathy may be incomplete due to mild manifestations or due to occurrence during ICU stay.

Before diagnosing SC2-GBS, it is crucial to exclude various differential diagnoses. These include previously existing neuropathy, critical ill myopathy, critical ill neuropathy, toxic neuropathy, or neuropathy or myopathy due to side effects of applied drugs. Lopinavir and tocilizumab have been reported to cause neuropathy $[8$, 9]. There are also reports indicating that chloroquine may induce neuropathy [10].

If GBS develops during immobilisation for artificial ventilation, diagnosing SC2-GBS becomes challenging [7]. In patients under artificial ventilation for COVID19, SC2-GBS should be considered if clinical neurologic exam suggests neuropathy and if patients cannot be weaned from the respirator. In this case, nerve conduction studies and investigations of the CSF should be initiated. Diagnosing SC2-GBS is crucial as appropriate treatment may improve the overall outcome of COVID19 patients [11].

In some cases, SC2-GBS develops before classical clinical manifestations of the infection [12] being explained by subclinical infection with the virus prior to onset of GBS or the incubation time of SARS-CoV-2, which is up to 14 days [7].
Though there are no prediction models for the outcome or the need of artificial ventilation in SC2-GBS patients available, there are indications that the outcome is poor if there are complications from hypercoagulability (stroke, pulmonary embolism) and if there are superinfections or sepsis.

Most of the studies included in this review did not specify if respiratory failure in SC2-GBS patients resulted from brainstem encephalitis, BFE, involvement of the respiratory muscles in GBS, from pneumonia ending up as acute, respiratory distress syndrome (ARDS), from pulmonary embolism, heart failure, or from mixtures of these conditions. Specifying the cause of respiratory failure however is crucial as treatment and outcome may differ significantly among these conditions.

\section{Conclusions}

SC2-GBS is most likely secondary to an immune reaction against SARS-CoV-2 since the virus has not been found in CSF of any SC2-GBS patient reported. SC2GBS occurs at any age. SC2-GBS does not differ from non-SC2-GBS regarding clinical presentation and treatment, but the outcome of SC2-GBS is worse compared to non-CS2-GBS patients. The prevalence/incidence of GBS most likely increased since the outbreak of the pandemic. Since there are no studies about the optimal treatment of SC2-GBS subtypes available, they should be treated empirically in the same way as non-SC2-GBS subtypes. Early diagnosis of SC2-GBS is warranted because if appropriate treatment is applied in due time, the overall outcome from the infection may improve.

\section{Abbreviations \\ AIDP: Acute, inflammatory, demyelinating polyneuropathy; AMAN: Acute, motor axonal neuropathy; AMSAN: Acute, motor and sensory, axonal neuropathy; ARDS: Acute, respiratory distress syndrome; BFE: Bickerstaff encephalitis; CNS: Central nervous system; CSF: Cerebrospinal fluid; GBS: Guillain-Barre syndrome; IVIG: Intravenous immunoglobulins; MFS: Miller- Fisher syndrome; PCB: Pharyngeal, cervical, and brachial variant; PNC: Polyneuritis cranialis; PNS: Peripheral nervous system; SC2-GBS : SARS- CoV-2-associated GBS}

\section{Acknowledgements \\ None}

\section{Authors' contributions}

JF: design, literature search, discussion, first draft, critical comments, FS: literature search, discussion, critical comments, final approval.

All authors have read and approved the manuscript and ensured that this is the case.

\section{Funding}

None received

\section{Availability of data and materials}

Not applicable

\section{Declarations}

Ethics approval and consent to participate Not applicable 


\section{Consent for publication}

Not applicable

\section{Competing interests}

The authors declare that they have no competing interests.

\section{Author details}

${ }^{1}$ Klinik Landstrasse, Messerli Institute, Postfach 20, 1180 Vienna, Austria. ${ }^{2}$ Disciplina de Neurociência. Universidade Federal de São Paulo/Escola Paulista de Medicina (UNIFESP/EPM), São Paulo, Brazil.

Received: 5 January 2021 Accepted: 23 April 2021

Published online: 04 May 2021

\section{References}

1. Munjal M, Das S, Chatterjee N, Setra AE, Govil D. Systemic involvement of novel coronavirus (COVID-19): a review of literature. Indian J Crit Care Med. 2020;24(7):565-9. https://doi.org/10.5005/jp-journals-10071-23498.

2. da Rosa MR, Francelino Silva Junior LC, Santos Santana FM, Farias de Oliveira T, Campos Alcântara R, Monteiro Arnozo G, et al. Clinical manifestations of COVID-19 in the general population: systematic review. Wien Klin Wochenschr. 2020:1-6. (in press)

3. Baig AM, Sanders EC. Potential neuroinvasive pathways of SARS-CoV-2: deciphering the spectrum of neurological deficit seen in coronavirus disease-2019 (COVID-19). J Med Virol. 2020;92(10):1845-57. https://doi.org/1 0.1002/jmv.26105.

4. Paliwal VK, Garg RK, Gupta A, Tejan N. Neuromuscular presentations in patients with COVID-19. Neurol Sci. 2020;41(11):3039-56. https://doi.org/10.1 007/s10072-020-04708-8.

5. Nguyen TP, Taylor RS. Guillain Barre syndrome. 2020 Nov 19. In: StatPearls [Internet]. Treasure Island (FL): StatPearls Publishing; 2021. Jan-. [last accessed $26^{\text {th }}$ February 2021].

6. Keddie S, Pakpoor J, Mousele C, Pipis M, Machado PM, Foster M, et al. Epidemiological and cohort study finds no association between COVID-19 and Guillain-Barré syndrome. Brain. 2020:awaa433. (in press)

7. Filosto M, Cotti Piccinelli S, Gazzina S, Foresti C, Frigeni B, Servalli MC, et al. Guillain-Barré syndrome and COVID-19: an observational multicentre study from two Italian hotspot regions. J Neurol Neurosurg Psychiatry. 2020; jnnp2020-324837.

8. Khanlou H, Valdes-Sueiras M, Farthing C. Peripheral neuropathy induced by lopinavir-saquinavir-ritonavir combination therapy in an HIV-infected patient. J Int Assoc Physicians AIDS Care (Chic). 2007;6:155.

9. Sugiura F, Kojima T, Oguchi T, Urata S, Yuzawa Y, Sakakibara A, et al. A case of peripheral neuropathy and skin ulcer in a patient with rheumatoid arthritis after a single infusion of tocilizumab. Mod Rheumatol. 2009;19(2): 199-203. https://doi.org/10.3109/s10165-008-0132-2.

10. Becerra-Cuñat JL, Coll-Cantí J, Gelpí-Mantius E, Ferrer-Avellí X, LozanoSánchez M, Millán-Torné M, et al. Chloroquine-induced myopathy and neuropathy: progressive tetraparesis with areflexia that simulates a polyradiculoneuropathy. Two case reports. Rev Neurol. 2003;36(6):523-6.

11. Dubey D, Kapotic M, Freeman M, Sawhney A, Rojas JC, Warnack W, et al. Factors contributing to delay in diagnosis of Guillain-Barré syndrome and impact on clinical outcome. Muscle Nerve. 2016;53(3):384-7. https://doi. org/10.1002/mus.24772.

12. Finsterer J, Scorza FA, Fiorini AC. SARS-CoV-2-associated guillain-barre syndrome in 62 patients. Eur J Neurol. 2020;28(1):e10-2. https://doi.org/1 0.1111/ene. 14544

\section{Publisher's Note}

Springer Nature remains neutral with regard to jurisdictional claims in published maps and institutional affiliations.

\section{Submit your manuscript to a SpringerOpen ${ }^{\circ}$ journal and benefit from:}

- Convenient online submission

- Rigorous peer review

- Open access: articles freely available online

- High visibility within the field

- Retaining the copyright to your article

Submit your next manuscript at $\boldsymbol{\nabla}$ springeropen.com 